\title{
BMJ Open Guidelines about physical activity and exercise to reduce cardiometabolic risk factors: protocol for a systematic review and critical appraisal
}

\author{
Madelin Siedler (D) , ${ }^{1}$ M Hassan Murad, ${ }^{2,3}$ Yngve Falck-Ytter, ${ }^{4}$ Philipp Dahm, ${ }^{5,6}$ \\ Reem A Mustafa, ${ }^{7,8}$ Shahnaz Sultan, ${ }^{9,10}$ Rebecca L Morgan ${ }^{8}$
}

To cite: Siedler M, Murad MH, Falck-Ytter Y, et al. Guidelines about physical activity and exercise to reduce cardiometabolic risk factors: protocol for a systematic review and critical appraisal. BMJ Open 2020;10:e032656. doi:10.1136/ bmjopen-2019-032656

- Prepublication history and additional material for this paper are available online. To view these files, please visit the journal online (http://dx.doi. org/10.1136/bmjopen-2019032656).

Received 28 June 2019 Revised 30 October 2019 Accepted 10 January 2020

Check for updates

(c) Author(s) (or their employer(s)) 2020. Re-use permitted under CC BY-NC. No commercial re-use. See rights and permissions. Published by BMJ.

For numbered affiliations see end of article.

Correspondence to

Dr M Hassan Murad;

murad.mohammad@mayo.edu

\section{ABSTRACT}

Introduction Physical activity can prevent a wide range of diseases, including highly prevalent conditions such as heart disease, diabetes and associated cardiometabolic disorders. Numerous guidelines for the prescription of physical activity and exercise to promote general health and prevent disease are released each year, but the quality of these guidelines is currently unknown. This systematic review and critical appraisal of physical activity and exercise guidelines aims to summarise the current status and quality of these guidelines to provide suggestions to improve the development of future guidelines in this area.

Methods and analysis We will conduct a systematic review of guidelines in Ovid MEDLINE(R) and Epub Ahead of Print, In-Process \& Other Non-Indexed Citations, and Daily, Ovid EMBASE, Ovid Cochrane Central Register of Controlled Trials, Ovid Cochrane Database of Systematic Reviews and Scopus databases published from database 2000 through 23 0ctober 2019, written in English for the use of physical activity and exercise for the prevention of cardiometabolic disease and related risk factors in otherwise healthy individuals. We will also search the grey literature for additional eligible documents. We will use the Appraisal of Guidelines for Research and Evaluation II tool to assess the quality of eligible recommendations from all included guidelines, as well as perform exploratory analyses on guideline development variables.

Ethics and dissemination As a protocol for the review and critical appraisal of published documents, no potential ethical considerations are discussed. The protocol will guide the development of the review, which will be disseminated to relevant journals for publication. PROSPERO registration number CRD42019126364

\section{INTRODUCTION}

\section{Statement of purpose}

Despite widespread recommendations for the use of physical activity as a preventive health measure, the quality of guidelines for physical activity and exercise prescription in the general public is currently unknown. Previously published reviews of guidelines
Strengths and limitations of this study

- A systematic search of the published literature using relevant key terms will be conducted in addition to a search of the grey literature.

- Each item will be independently screened by two authors, and if included, will undergo independent quality appraisal by two authors.

- Both Appraisal of Guidelines for Research and Evaluation II and Institute of Medicine criteria will be used to assess each included document.

- Additional sensitivity analyses will report on any effects of guideline attributes, such as region of origin and development methodology used, on quality.

- Limitations include the fact that other physical activity guidance documents intended for the prevention of cancer, osteoporosis, falls/injuries and other risks were excluded from the scope of the present review, and that the grey literature search is primarily composed of professional societies within North America, specifically the USA.

for physical activity in specific clinical populations such as patients with osteoporosis $^{12}$ and schizophrenia ${ }^{2}$ reported a high variability in the quality of guidelines. Of specific concern were lack of specificity for the implementation of recommendations and lack of adequate stakeholder involvement throughout the guideline development process. However, to our knowledge, there are no systematic critical appraisals of physical activity guidelines written for the general healthy population, including all age and demographic groups. The development of such an appraisal within a specific field of guidance, such as physical activity and exercise, provides an overview of the currently available guidance while serving as a platform for the development of more rigorous and transparent processes of guideline development and identifying areas in need of improvement. 


\section{Description of the condition}

Heart disease, diabetes and ischaemic stroke are among the top ten leading causes of death worldwide, with particularly high rates of death occurring in middle-income and high-income countries. ${ }^{3}$ In the USA, these three conditions caused more than 877000 deaths over the course of a recent year. ${ }^{4}$ These conditions are often accompanied by a set of comorbid disorders and cardiometabolic risk factors, including obesity, hypertension and dyslipidemia, and their incidence and prevalence continue to rise around the globe. ${ }^{5}$

\section{Description of the intervention}

Physical activity is the use of bodily movement produced by the contraction of skeletal muscles and resulting in energy expenditure. Physical activity is a general term that includes spontaneous and informal forms of movement such as those required for daily living, whereas exercise often refers to a subtype of physical activity that is structured and purposeful. ${ }^{6}$ For the purposes of this review, the term 'physical activity' will be used to describe interventions aiming to increase energy expenditure through bodily movement, whether planned or informal.

According to the U.S. Centers for Disease Control and Prevention, sufficient amounts of physical activity could prevent as many as 1 in every 10 premature deaths and save up to US $\$ 117$ billion in annual healthcare costs. ${ }^{7}$ In addition to its major role in preventing cardiovascular disease, diabetes and 13 types of cancer, physical activity bestows benefits across the lifespan, improving aerobic fitness, bone health and academic performance in children; preventing overweight and obesity and improving mental health in adults; and preventing injury and cognitive decline and promoting late-life independence in older adults. $^{7-10}$

Physical activity is also a cost-effective behaviour for the prevention of chronic disease. ${ }^{11}$ A 2016 study using medical expenditure data from over 26000 adults in the USA with and without cardiovascular disease showed that physical activity was associated with lower healthcare expenditures regardless of cardiovascular disease or risk factors, with 'optimal' levels of physical activity (over $30 \mathrm{~min}$ of moderate to vigorous exercise, five times per week) creating an average savings of US\$2500 in healthcare costs annually. ${ }^{12}$ Optimal levels of physical activity were also associated with significantly lower rates of healthcare resource utilisation, including emergency department visits, outpatient visits, and prescription use. An earlier survey of 8000 older adults found that physical inactivity, overweight and obesity were associated with nearly one-quarter $(23 \%)$ of healthcare plan costs. ${ }^{13}$

In addition to its reported health benefits, physical activity is a conservative and non-invasive intervention that can be implemented and tailored to the specific needs and abilities of individuals across their lifespan. This is demonstrated in the assessment of a person's health, needs and abilities when designing a physical activity programme, including considerations and contraindications for special populations such as children, the elderly, and individuals with pre-existing conditions. The performance of such a needs and safety assessment is a key focus of commonly used personal training certifications in the USA. ${ }^{14-16}$

The use of general recommendations for physical activity to promote health have existed since as early as 3000 BC. ${ }^{17}$ In their modern form, guidelines for the use of physical activity in the promotion of general health and the prevention of disease have existed since at least 1996. ${ }^{18}$ Physical activity guidelines contain recommendations to inform healthcare professionals and the general population about optimal modalities, frequency, duration, and intensity of physical activity and exercise in order to prevent deleterious health outcomes.

\section{How the intervention might work}

Physical activity leads to a number of physiological adaptations in the body that decrease the risk of cardiometabolic disease. In this regard, the beneficial effects of physical activity and exercise include improved body composition and decreased visceral adiposity; improved lipoprotein profiles, insulin sensitivity and endothelial function; and reductions in resting blood pressure, resting heart rate and systemic inflammation. ${ }^{11}$ More specifically, aerobic types of physical activity which require a sustained increase in heart rate are associated with beneficial adaptations including increased stroke volume and cardiac output, reduced resting heart rate, increased mitochondrial and capillary density, and improved body composition through reductions in fat mass. ${ }^{19-21}$ Anaerobic types of activity such as resistance training lead to reductions in resting blood pressure via decreased peripheral vascular resistance as well as increases in insulin sensitivity and improvements in body composition and resting metabolic rate by promoting the accretion of metabolically active lean tissue. ${ }^{192122}$

Physical activity guidelines inform the public and providers on the optimal modality, frequency, duration and intensity of exercise for general health and disease prevention, as well as communicate the expected health outcomes related to physical activity. In this way, physical activity guidelines have the potential to improve physical activity behaviours, leading to improved health outcomes. However, in order for a guideline to have adequate uptake and utilisation, it is important that it is developed with adequate rigour, contains recommendations that are clearly articulated and provides information or tools for practical implementation.

\section{OBJECTIVES}

This review aims to answer the following question: what is the quality of guidelines written for the use of exercise and physical activity to promote health and reduce the risk of cardiometabolic disease in the general population, including adults and the elderly, children and adolescents, and pregnant women? 


\section{METHODS AND ANALYSIS}

\section{Types of documents}

We will only include guidance documents that contain recommendations such as guidelines, consensus statements and position statements written in English and published by a professional organisation, society, nonprofit or governmental organisation, or ad hoc task force or working group comprising professionals in the field. Documents published by for-profit entities such as businesses will be excluded.

\section{Types of populations}

Guidance documents written for use in the healthy general population or for the primary prevention of the following cardiometabolic conditions will be included:

- Heart disease.

- Hypertension.

- Dyslipidemia.

- Stroke.

- Diabetes mellitus and dysglycemia.

Physical activity guidelines written for the management or prevention of overweight and obesity separate from the management or secondary prevention of the aforementioned related cardiometabolic disorders will also be included. Guidelines written for the use of physical activity in the management or secondary prevention of other disease will not be included. For the purposes of this review, the general population includes children/ youth, adults, pregnant women and the elderly. Additional sensitivity analyses will be performed to report on any differences in quality or clarity between recommendations targeted towards these subpopulations.

\section{Types of interventions}

Any recommendation which is identified from an eligible guidance document and which involves the use of physical activity or exercise including aerobic, musclestrengthening/resistance-training, balance or flexibility training, or unstructured play will be considered. Physical activity and exercise interventions of any duration, intensity, modality or programme length will be considered eligible for analysis.

\section{Types of outcome assessments}

The primary outcomes of this review will be to report on the overall quality of included physical activity and exercise guidelines for primary or secondary prevention of cardiometabolic disease, obesity, and overweight as assessed using the Appraisal of Guidelines for Research and Evaluation (AGREE) II tool. ${ }^{23}$ The AGREE II tool was developed as a way to assess the methodological quality of guidelines in a common and transparent manner. It includes six domains which allow the reader to assess a guideline's scope and purpose, stakeholder involvement, rigour of development, clarity of presentation, applicability and editorial independence. Analysis of guidelines using the AGREE II tool allows for the identification of areas in which an individual guideline, guideline developer, or field of guidance excels or requires improvement. The AGREE II tool has been used to critically appraise the quality of guidelines for physical activity and exercise in clinical populations as well as the quality of nutritional guidelines. ${ }^{1224}$

In addition, each extracted recommendation will be assessed to determine the clarity of recommendation. Recommendations will be assigned a score of $0-4$ by two independent reviewers based on the amount of information provided for the practical application of the recommendation by end-users. Sensitivity analyses will be performed to determine whether the quality of guidelines as assessed by the AGREE II tool is affected by a number of variables, such as year of publication, adherence to guideline development methodology and region of origin.

\section{Search methods for identification of studies}

We will conduct a search of the literature in two stages to capture guidelines in peer-reviewed and grey domains. Literature search results will be presented in a Preferred Reporting Items in Systematic reviews and Meta-Analyses (PRISMA) flowchart.

\section{Electronic searches}

A comprehensive search of several databases from 2000 to 23 October 2019, in the English language will be conducted. The databases to be searched include Ovid MEDLINE(R) and Epub Ahead of Print, In-Process \& Other Non-Indexed Citations, and Daily, Ovid EMBASE, Ovid Cochrane Central Register of Controlled Trials, Ovid Cochrane Database of Systematic Reviews and Scopus. The search strategy was designed and will be conducted by an experienced librarian with input from the study's principle investigator. Controlled vocabulary supplemented with keywords will be used to search for physical activity guidelines. The detailed strategy will be presented in the final manuscript. General terms that were used to guide the development of the search strategy are presented in table 1.

Table 1 General terms compiled to guide development of search strategy

\begin{tabular}{|c|c|}
\hline Section & Terms \\
\hline $\begin{array}{l}\text { Terms } \\
\text { related to } \\
\text { activity }\end{array}$ & $\begin{array}{l}\text { "physical activity," OR "exercise" OR "physical } \\
\text { fitness" OR "aerobic exercise," OR "resistance } \\
\text { training," OR "strength training" OR "balance" } \\
\text { OR "flexibility" }\end{array}$ \\
\hline $\begin{array}{l}\text { MeSH terms } \\
\text { related to } \\
\text { outcomes of } \\
\text { interest }\end{array}$ & $\begin{array}{l}\text { "physical fitness" OR "cardiorespiratory } \\
\text { fitness" OR "glucose metabolism disorders" } \\
\text { OR "metabolic syndrome" OR "obesity" OR } \\
\text { "heart diseases" or "vascular diseases" OR } \\
\text { "mortality" OR "child" OR "adult, aged" }\end{array}$ \\
\hline $\begin{array}{l}\text { Terms of } \\
\text { article type }\end{array}$ & "guideline*" OR "position stand" \\
\hline
\end{tabular}




\section{Other resources}

Second, we will search the Web for guidelines produced by a predefined list of federal agencies, professional societies and organisations related to physical fitness, activity, exercise and sports medicine in order to capture internally published guidelines. A list of all institutions included in the manual Web search can be found in online supplementary appendix A. The official web site of each organisation will be studied in order to locate published guidance documents eligible for appraisal.

\section{DATA COLLECTION AND ANALYSIS \\ Selection of studies}

Literature identified in the searches will undergo an independent dual review screening process. Two reviewers (MS, MHM, YF-Y, RLM) will independently and in duplicate screen each title and abstract to determine whether the article meets eligibility for full-text screening. To be deemed eligible for inclusion, titles and abstracts must be guideline or guidance documents written in English guidelines for the use of physical activity and/or exercise in the general public (apparently healthy individuals of all demographics, including children, elderly, and adults, including pregnant women) for general health, the primary prevention of cardiometabolic disease (heart attack, stroke, diabetes, as well as prevention of CVD risk factors (eg hypertension, dyslipidemia)), or the prevention or management of overweight and obesity. Titles and abstracts identified as eligible by at least one reviewer will be included for full-text screening. Full texts will be reviewed in the same fashion by two reviewers using standardised eligibility criteria. In the case of disagreement, the article will be reviewed by a third reviewer to reach consensus. Results will be presented using the PRISMA flowchart (online supplementary appendix B) ${ }^{25}$

\section{Data extraction and management}

All authors will complete online training for the AGREE II tool. A standardised and pilot-tested data extraction form (online supplementary appendix C) will be used to collect information on (1) name of organisation(s); (2) publication date; (3) region of origin; (4) guideline development method (eg Grading of Recommendations Assessment, Development and Evaluation (GRADE) or other); (5) whether a systematic review was used to inform recommendations; (6) whether the use of patient representation is reported; (7) number of total recommendations; (8) number of recommendations related to aerobic activity, resistance/muscle- and/or bone-strengthening activity, balance/flexibility or unstructured play; (9) the clarity of recommendations within each of these modalities and (10) number of recommendations targeted to specific age groups or populations, and to whom (adults; children/adolescents; older adults; pregnant/postpartum women). Two reviewers will independently extract relevant information from eligible full-text guidelines.

\section{Unit of analysis}

The primary unit of analysis will be individual eligible guidance documents which include one or more recommendations for the use of aerobic, bone- or muscle-strengthening, flexibility or balance training, or unstructured play for the promotion of general health or for the primary prevention of cardiometabolic disease and related risk factors as described previously. The average of two reviewers' AGREE II scores within each domain will be used to analyse the quality of individual guidelines and to compare them to one another based on extracted guideline characteristics such as region or year of publication.

\section{Assessment of agreement}

We will evaluate agreement between the two reviewers on the six domains of the AGREE II tool using appropriate statistical measures.

\section{Additional analysis}

In addition, we will conduct sensitivity analyses to determine if overall guideline quality score as assessed with the AGREE II tool is affected by the following known variables:

- Year of publication.

- Country/region of publishing body.

- Type of developing institution.

- Governmental agency/public institution.

- Professional society/organisation.

- Other non-profit.

- Ad hoc/independent working group.

- Adherence to a formal guideline development methodology (eg, GRADE).

- Adherence to the National Academy of Sciences, Engineering, \& Medicine's standards for developing trustworthy clinical practice guidelines. ${ }^{26}$

We hypothesise that eligible documents with the following attributes will achieve higher AGREE II scores:

- Adherence to the National Academy of Sciences, Engineering, \& Medicine's standards for developing trustworthy clinical practice guidelines.

- Later year of publication.

For guidance documents with multiple recommendations, we will also assess each individual recommendation's clarity of presentation by determining whether the recommendations had sufficient specificity to allow end-users to replicate and implement the recommended action. For an individual recommendation to achieve a high rating, it needs to describe the recommended frequency (eg three times a week), duration (eg total minutes per session or week) and intensity (eg vigorous vs moderate) of the activity, as well as give specific examples of the modality within the text of the document. We hypothesise that recommendations for aerobic activity will be rated as having higher clarity of presentation than recommendations for bone- or muscle-strengthening, balance and flexibility, or unstructured play. 


\section{Patient and public involvement}

Patients will not be involved in the development of this review.

\section{DISCUSSION}

Given its numerous reported health benefits, potential to create cost savings and reduce resource utilisation, and applicability as a preventive health intervention to almost every individual, physical activity is an important topic for evidence-based health guidelines. Healthcare providers, fitness practitioners and the general public represent a vast and diverse group of potential stakeholders, including end-users, of such guidelines. Evidence-based guidelines that provide recommendations for the comparative effectiveness of optimal dosages and modalities of physical activity for the general population are therefore needed and highly valuable; however, the level of quality and rigour within these currently available guidelines has not been studied.

To our knowledge, there are no published quality appraisals of guidelines for the use of physical activity in the general public, including all age and demographic groups. A limited number of published appraisals of physical activity guidance in clinical populations, however, offers some insight to the quality and consistency of guidelines relating to physical activity. A 2011 review found that out of 12 recommendations for the use of physical activity to reduce the risk of developing cardiovascular disease in patients with schizophrenia, only one met the reviewers' quality standards. Noted issues included lack of a formal structure for rating the quality of evidence, lack of involvement of key stakeholders and low specificity of the recommendations themselves. A 2016 review of guidelines for physical activity in individuals with osteoporosis found that out of the 19 guidelines studied, only four (21\%) were deemed high-quality, meaning that the guideline obtained a score of at least $60 \%$ on at least five of the six quality domains. Identified issues included lack of clinical applicability, problems with conflict of interest disclosure, and lack of specificity in terms of activity dosage, progression and contraindications. ${ }^{1}$

A cursory review of physical activity guidelines developed for the general public suggests that while many of these guidelines are based on a systematic review of the evidence and use a formal grading system, the role of patient (end-user) perspectives in the guideline development process is not immediately clear. ${ }^{27}{ }^{28}$ Furthermore, some recommendations are left unspecific or ambiguous, making the communication and implementation of these recommendations difficult. Reliable and valid instruments such as the AGREE II tool exist to guide the critical appraise of the quality of published guidelines. ${ }^{23} \mathrm{~A}$ critical appraisal of these guidelines using the AGREE II criteria will allow us to summarise the strengths and shortcomings of currently available guidelines and provide suggestions for future research. Analyses within this study will inform recommendations and future directions for the development and dissemination of physical activity, fitness and exercise-related guidelines that meet standards for quality and rigour. As such, the study will identify critical opportunities for organisations, researchers and practitioners in the field to begin to fill these gaps and continue to move the field further in the direction of evidence-based practice.

\section{Author affiliations}

${ }^{1}$ Physical Education and Exercise Science, University of South Florida, Tampa, Florida, USA

${ }^{2}$ Evidence-Based Practice Center, Mayo Clinic, Rochester, Minnesota, USA ${ }^{3}$ Robert D and Patricia E Kern Center for the Science of Health Care Delivery, Mayo Clinic, Rochester, Minnesota, USA

${ }^{4}$ Gastroenterology, Case Western Reserve University, Cleveland, Ohio, USA

5Urology, Minneapolis VA Health Care System, Minneapolis, Minnesota, USA

${ }^{6}$ Urology, University of Minnesota, Minneapolis, Minnesota, USA

${ }^{7}$ Internal Medicine, University of Kansas Medical Center, Kansas City, Kansas, USA

${ }^{8}$ Health Research Methods, Evidence and Impact, McMaster University, Hamilton, Ontario, Canada

${ }^{9}$ Gastroenterology, Minneapolis VA Health Care System, Minneapolis, Minnesota, USA

${ }^{10}$ Gastroenterology, University of Minnesota, Minneapolis, Minnesota, USA

Contributors MS is the guarantor and drafted the manuscript. All authors contributed to the development of selection criteria and additional methodological considerations. MS developed the search strategy. RLM and MHM provided expertise on systematic review and guideline quality appraisal methods. MHM, YF-Y, PD, RAM, SS and RLM provided feedback and approved the final manuscript.

Funding The authors have not declared a specific grant for this research from any funding agency in the public, commercial or not-for-profit sectors.

Competing interests All authors disclose involvement with the U.S. GRADE Network and the Evidence Foundation. MS is a fellow of the Evidence Foundation and receives direct financial support.

Patient consent for publication Not required.

Provenance and peer review Not commissioned; externally peer reviewed.

Open access This is an open access article distributed in accordance with the Creative Commons Attribution Non Commercial (CC BY-NC 4.0) license, which permits others to distribute, remix, adapt, build upon this work non-commercially, and license their derivative works on different terms, provided the original work is properly cited, appropriate credit is given, any changes made indicated, and the use is non-commercial. See: http://creativecommons.org/licenses/by-nc/4.0/.

ORCID iD

Madelin Siedler http://orcid.org/0000-0002-2845-8174

\section{REFERENCES}

1 Armstrong JJ, Rodrigues IB, Wasiuta T, et al. Quality assessment of osteoporosis clinical practice guidelines for physical activity and safe movement: an agree II appraisal. Arch Osteoporos 2016;11.

2 Vancampfort D, Sweers K, Probst M, et al. Quality assessment of physical activity recommendations within clinical practice guidelines for the prevention and treatment of cardio-metabolic risk factors in people with schizophrenia. Community Ment Health J 2011;47:703-10.

3 World Health Organization. The top 10 causes of death, 2018. Available: https://www.who.int/en/news-room/fact-sheets/detail/thetop-10-causes-of-death [Accessed Apr 2019].

4 Centers for Disease Control and Prevention. Leading causes of death, 2017. Available: https://www.cdc.gov/nchs/fastats/leadingcauses-of-death.htm [Accessed Oct 2019].

5 Ndisang JF, Rastogi S. Cardiometabolic diseases and related complications: current status and future perspective. Biomed Res Int 2013;2013:1-3.

6 World Health Organization. Diet, physical activity and health, 2019. Available: https://www.who.int/dietphysicalactivity/pa/en/ [Accessed Apr 2019]. 
7 Centers for Disease Control and Prevention. About physical activity: why it matters, 2019. Available: https://www.cdc.gov/ physicalactivity/about-physical-activity/why-it-matters.html

8 Varghese T, Schultz WM, McCue AA, et al. Physical activity in the prevention of coronary heart disease: implications for the clinician. Heart 2016;102:904-9.

9 Garber CE, Blissmer B, Deschenes MR, et al. American College of sports medicine position stand. quantity and quality of exercise for developing and maintaining cardiorespiratory, musculoskeletal and neuromotor fitness in apparently healthy adults: guidance for prescribing exercise. Med Sci Sports Exerc 2011;43:1334-59.

10 Kist C, Gier A, Tucker J, et al. Physical activity in clinical pediatric weight management programs: current practices and recommendations. Clin Pediatr 2016;55:1219-29.

11 Warburton DER, Bredin SSD. Health benefits of physical activity: a systematic review of current systematic reviews. Curr Opin Cardiol 2017;32:541-56.

12 Valero-Elizondo J, Salami JA, Osondu CU, et al. Economic Impact of Moderate-Vigorous Physical Activity Among Those With and Without Established Cardiovascular Disease: 2012 Medical Expenditure Panel Survey. J Am Heart Assoc 2016;5.

13 Anderson LH, Martinson BC, Crain AL, et al. Health care charges associated with physical inactivity, overweight, and obesity. Prev Chronic Dis 2005;2:A09.

14 American Council on Exercise. Appendix B: exam content outline, 2016. Available: https://acewebcontent.azureedge.net/assets/ certification/pdfs/CPT-Exam-Content-Outline.pdf [Accessed Apr 2019].

15 American College of Sports Medicine. ACSM personal trainer job task analysis, 2017. Available: https://www.acsm.org/docs/defaultsource/certification-documents/cpt/2017-acsm-cpt-jta-full-final-1. pdf?sfvrsn=dc02638an_10 [Accessed Apr 2019].

16 National Strength and Conditioning Association. NSCA-Certified personal trainer exam description, 2018. Available: https://www.nsca. com/certification/nsca-cpt/nsca-cpt-exam-description [Accessed Apr 2019].

17 Shalala DE. Physical activity and health: a report of the surgeon General executive summary Centers for Disease Control and Prevention; 1996.
18 Troiano RP. History of physical activity recommendations and guidelines for Americans. Available: https://health.gov/paguidelines/ second-edition/meetings/1/History-of-Physical-ActivityRecommendations-and-Guidelines-for-Americans.pdf [Accessed 20 Oct 2019].

19 Smith DL, Fernhall B. Advanced cardiovascular exercise physiology. Champaign, IL: Human Kinetics, 2011.

20 Hellsten Y, Nyberg M. Cardiovascular adaptations to exercise training. Compr Physiol 2015;6:1-32.

21 Haff G, Triplett NT, and National Strength \& Conditioning Association. Essentials of strength training and conditioning. Fourth edition. Champaign, IL: Human Kinetics, 2016.

22 Winett RA, Carpinelli RN. Potential health-related benefits of resistance training. Prev Med 2001;33:503-13.

23 Brouwers MC, Kho ME, Browman GP, et al. Agree II: advancing Guideline development, reporting and evaluation in health care. Can Med Assoc J 2010;182:E839-42.

24 Rabassa M, Garcia-Ribera Ruiz S, Solà I, et al. Nutrition guidelines vary widely in methodological quality: an overview of reviews. J Clin Epidemiol 2018;104:62-72.

25 Liberati A, Altman DG, Tetzlaff J, et al. The PRISMA statement for reporting systematic reviews and meta-analyses of studies that evaluate healthcare interventions: explanation and elaboration. BMJ 2009;339:b2700.

26 Institute of Medicine (US) Committee on Standards for Developing Trustworthy Clinical Practice Guidelines. Standards for developing trustworthy clinical practice guidelines. Washington, DC: National Academies Press, 2011. https://www.ncbi.nlm.nih.gov/books/ NBK209539/

27 Arnett DK, Blumenthal RS, Albert MA, et al. 2019 ACC/AHA guideline on the primary prevention of cardiovascular disease: a report of the American College of Cardiology/American heart association Task force on clinical practice guidelines. Circulation 2019;140:e596-646.

28 Anderson TJ, Grégoire J, Pearson GJ, et al. 2016 Canadian cardiovascular Society guidelines for the management of dyslipidemia for the prevention of cardiovascular disease in the adult. Can J Cardiol 2016;32:1263-82. 